\title{
Atomic Structure and Bonding of Boron-Induced Reconstructions on Sil001)
}

\section{Citation}

Wang, Yajun, Robert J. Hamers, and Efthimios Kaxiras. 1995. "Atomic Structure and Bonding of Boron-Induced Reconstructions on Si(001)." Physical Review Letters 74 (3): 403-6. https:// doi.org/10.1103/physrevlett.74.403.

\section{Permanent link}

http://nrs.harvard.edu/urn-3:HUL.InstRepos:41384108

\section{Terms of Use}

This article was downloaded from Harvard University's DASH repository, and is made available under the terms and conditions applicable to Other Posted Material, as set forth at http:// nrs.harvard.edu/urn-3:HUL.InstRepos:dash.current.terms-of-use\#LAA

\section{Share Your Story}

The Harvard community has made this article openly available.

Please share how this access benefits you. Submit a story.

Accessibility 


\section{Atomic Structure and Bonding of Boron- Induced Reconstructions on $\mathrm{Si}(001)$}

Yajun Wang and Robert J. Hamers*

Department of Chemistry, University of Wisconsin-Madison

1101 University Avenue, Madison, WI 53706

E-mail: Hamers@BERT.CHEM.WISC.EDU

and

Efthimios Kaxiras

Department of Physics and Division of Applied Science

Harvard University, Cambridge, MA 02138

Suggested PACS numbers: 73.20.-r, 68.55.Ln, 61.16.-Ch, 61.72.Tt

Abstract

Scanning tunneling microscopy and tunneling spectroscopy have been used to investigate the local structural and electronic properties of boron-induced reconstructions on $\mathrm{Si}(001)$. Thermal decomposition of diborane produces three ordered reconstructions, which arise from ordered arrangements of three structural subunits, with a local boron coverage of $1 / 2$ monolayer. A structural model is proposed which accounts for the observed STM features. The principal structural subunit is shown to be an ordered arrangement of four boron atoms at substitutional sites in the first bulk-like silicon layer, which is then capped with ordered arrangements of silicon dimers and dimer vacancies.

*Author to whom correspondence should be addressed. 
Doping of semiconductor structures is a strongly non-equilibrium process involving a complex interplay of surface structure and chemical kinetics. Recent investigations have shown that the interfacial chemistry of boron with silicon permits the fabrication of narrow, non-equilibrium two-dimensional layers of highly-doped silicon with high structural and electrical quality. ${ }^{1-8}$ The growth of such non-equilibrium structures by chemical techniques presents a novel method for preparation of nanometer-scale electronic devices based on self-limiting interfacial chemistry. In this study, we report the first scanning tunneling microscopy (STM) studies of boron-induced reconstructions and the formation of $\delta$ doped layers on $\mathrm{Si}(001)$. Our results show that boron interacts with $\mathrm{Si}(001)$ to produce several complex, ordered reconstructions which can be described as arrangements of three basic structural subunits. A detailed analysis of the STM results, in conjunction with other electrical and chemical probes of the surface, provides new insight into the structure and bonding within boron $\delta$-doped layers.

$\mathrm{Si}(001)$ samples (Wacker) were cleaned by annealing at 1425 Kelvin in ultrahigh vacuum. These were imaged with the STM to ensure a clean, well-ordered starting surface and were then typically exposed to $1 \%$ diborane in $\mathrm{He}$ (5000 Langmuirs, where 1 Langmuir=10-6 torr sec) or pure decaborane (0.1-1 Langmuir) at 815 Kelvin followed by annealing at 1000 Kelvin for 90 seconds. Both diborane and decaborane produced similar structures, but images shown here were obtained using diborane.

While the clean $\mathrm{Si}(001)$ surface has large, flat terraces, fig. 1 shows that exposure to diborane at elevated temperatures produces islands atop the terraces. These islands as well as many smaller regions of the substrate (13 regions in fig. 1, labeled "RS") exhibit a complex boron- 
induced surface reconstruction. The boron-reconstructed regions are separated by regions of clean $\operatorname{Si}(001)-(2 \times 1)$, leading to a striking spatial segregation of boron into regions of high concentration (evidenced by local reconstructions) and regions of nearly clean (undoped) $\mathrm{Si}$.

Figure 2 shows high-resolution STM images of the boron-induced reconstructions. In fig. $2 a$, small regions showing $c(4 \times 4)$ symmetry (square unit cell rotated $45^{\circ}$, labeled " $\alpha$ ") and (4x4) symmetry (square unit cell, labeled " $\gamma$ ") can be observed. Additionally, some regions showing the $(2 \times 1)$ reconstruction of the "clean" $\mathrm{Si}(001)$ surface can be observed (rectangular unit cell, labeled "Si"). The local electronic structure of these reconstructions is revealed by comparing images probing the occupied and unoccupied electronic states of a single area, as shown in fig. $2 b$ (occupied. states, $-2 \mathrm{~V}$ sample bias) and $2 \mathrm{c}$ (unoccupied states, $+2 \mathrm{~V}$ bias). In fig. $2 \mathrm{~b}$ and $2 c$, the surface again shows primarily the $c(4 \times 4)$ reconstruction, with a few unit cells of $(4 \times 4)$ symmetry. Additionally, small regions exhibiting yet another reconstruction of $c(4 \times 4)$ symmetry can be observed, labeled $" \beta "$. For clarity, we will refer to the most common structure of $c(4 \times 4)$ symmetry as $" \alpha-c(4 \times 4) "$, the less common $c(4 \times 4)$ reconstruction as $" \beta$ $c(4 \times 4) "$, and the $(4 \times 4)$ reconstruction as " $\gamma-(4 \times 4) "$.

Close inspection shows that all three boron-induced reconstructions share common structural features. We consider first the most common, the $\alpha-c(4 \times 4)$ reconstruction. At negative sample bias each unit cell contains two equivalent oval-shaped protrusions and a single, unique oval protrusion which under these conditions appears $0.35 \AA$ higher. Although the highest-symmetry unit cell can be described as a single high oval protrusion bounded on each side by a single lower oval, examination of the top and bottom edges of the island in fig. $2 b$ and $2 c$ shows that the edges 
of reconstructed areas always terminate with a pair of the lower protrusions. Thus, the $\alpha-c(4 \times 4)$ reconstruction is best described in terms of two structural building blocks: a pair of two equivalent oval-shape protrusions, labeled " $\mathrm{A}$ " in fig. 2, and a single unique oval-shaped protrusion, labeled "B" in fig. 2. This structure consisting of one "A" subunit and one "B" subunit repeats every $4 \mathrm{a}_{0}$ (where $\mathrm{a}_{0}=3.84 \AA$ ) along the $<1 \overline{1} 0>$ direction and is ordered between adjacent rows in $c(4 \times 4)$ symmetry as indicated in fig. 2. At positive bias, fig. 2c shows that the "B" feature splits into two well-resolved nearly circular protrusions, and the two oval-shape protrusions constituting the " $\mathrm{A}$ " feature now appear $0.4 \AA$ higher than the "B" feature, but otherwise unchanged in appearance. The changes in appearance of the "B" features as a function of bias are very similar to those exhibited by dimers of the clean $\mathrm{Si}(001)$ surface, suggesting a common origin.

The $\gamma-(4 \times 4)$ reconstruction is nearly identical to the $\alpha-c(4 \times 4)$, except that half the "B" features appear to be replaced by vacancies.

Finally, the $\beta-c(4 \times 4)$ reconstruction visible at lower right in fig. $2 b$ and $2 c$ arises from an ordered arrangement of the "A" features alone. Within each $\beta-c(4 \times 4)$ unit cell, the two ovals are separated along the $<1 \overline{1} 0\rangle$ direction by a distance alternating between $5.0 \AA$ and $10.4 \AA$ (compared with $\mathrm{a}_{0}=3.84 \AA$ ), such that the ovals slow a clear grouping into pairs. The shape, grouping, and apparent height of the oval protrusions in the $\beta-c(4 \times 4)$ reconstruction are nearly identical to those of the "lower" oval protrusions in the $\alpha-c(4 \times 4)$ and $\beta-c(4 \times 4)$ reconstructions, indicating that the "A" subunit is common to all three.

Further insight into is provided by STM measurements which show that exposing $\mathrm{Si}(001)$ to much smaller amounts of $\mathrm{B}_{2} \mathrm{H}_{6}(50$ Langmuirs) 
produces only the "A" features, while at intermediate exposures (100-500 Langmuirs) these group together (alone and with the "B" features) to form the various ordered reconstructions. These results confirm that the "A" feature is the primary boron-induced surface feature and that this same feature is contained in all three boron-induced reconstructions. Additionally, the features reported here are not produced at sample temperatures below $755 \mathrm{~K}$, indicating substantial atomic rearrangement. To determine the local boron coverage within the reconstructed regions, Auger electron spectra were obtained on a number of samples exposed to diborane. The $\mathrm{B} / \mathrm{Si}$ peak ratio of the $\mathrm{B} / \mathrm{Si}(001)$ samples was compared with that for the $\operatorname{Si}(111)-(\sqrt{3} \times \sqrt{3}) B$ reconstruction, which arises at $1 / 3$ monolayer $B, 9,10$ and corrections were applied based on the fractional area occupied by the reconstructions measured in-situ with STM. These measurements showed that the B coverage within the reconstructed $\mathrm{B} / \mathrm{Si}(001)$ regions was $0.56 \pm 0.05$ monolayer, or a coverage of $1 / 2$, in agreement with previous studies of $\delta$-doped $\mathrm{B} / \mathrm{Si}(001) .{ }^{3-8,11,12}$

The electronic properties of these layers were characterized in two ways. Figure 3 shows the results of local tunneling spectra at two locations within the $\alpha-c(4 \times 4)$ regions. Spectra measured over the bright "B" subunits show a bandgap of approximately $0.7 \mathrm{eV}$ with the Fermi level $\left(E_{F}\right)$ in the lower part of the surface-state gap, similar to spectra obtained on dimers of clean $\mathrm{Si}(001)$. In contrast, spectra measured directly above the "A" features show a high current (a high state density) just below $E_{F}$, with a $0.5 \mathrm{eV}$ gap above. This high state density just below $\mathrm{E}_{F}$ is what one would expect if the "A" features act as electron acceptors (p-type dopants) pinning the Fermi level near the valence band edge. Local surface photovoltage (SPV) measurements of these layers on n-type substrates 
further showed that when measured over the clean $\mathrm{Si}$ regions the local SPV, normally positive on n-type sample,s becomes more negative and shows local p-type doping as the reconstructed area increases.

Figure 4 shows a structural model we have developed for the boroninduced reconstructions. The STM studies show that only the "A" feature is observed at low coverage, and this feature alone is common to all three boron-induced reconstructions. The 0.5 monolayer boron coverage requires that each " $\mathrm{A}$ " feature results from four boron atoms, while p-type doping behavior and the high temperature required to form the reconstructions suggest a substitutional location. The STM images directly reveal the symmetry of the "A" features with respect to the $\mathrm{Si}(001)$ lattice, strongly constraining the possible boron positions. Finally, previous studies $^{2,3,7,8}$ have shown that epitaxial silicon can be grown atop these $\delta$ doped layers at low temperatures, so that any proposed model must allow for this possibility. Based on all these observations, we propose that the "A" features arise from $\mathrm{Si}$ dimers bonded above a $\mathrm{Si}(001)$ surface in which half the $\mathrm{Si}$ atoms in the first bulk-like layer are replaced by boron at the positions indicated in fig. 4 , and we propose the two oval-shaped features within each " $A$ " subunit arise from electronic states associated with these "boron-bonded" dimers and their Si-B bonds, as discussed below.

The "B" features show strong changes in appearance with applied bias that are identical to those observed for $\mathrm{Si}=\mathrm{Si}$ dimers of the clean surface, and the tunneling I-V spectra are also very similar. Likewise, the bonding location of feature "B" is also consistent with its interpretation as a $\mathrm{Si}=\mathrm{Si}$ dimer. Thus, we propose that the "B" feature arises from a $\mathrm{Si}=\mathrm{Si}$ dimer bonded to four second-layer $\mathrm{Si}$ atoms in a local environment similar to that of the clean $\mathrm{Si}(001)$ surface. 
With our proposed model, all three reconstructions can be described as ordered combinations of the "A" and "B" structural subunits together with dimer vacancies, and all three have $1 / 2$ monolayer boron coverage. The common $\alpha-c(4 \times 4)$ reconstruction arises from one "A" subunit and one "B" subunit in each unit cell, as depicted in fig. 4. The $\beta-c(4 \times 4)$ reconstruction arises from one " $\mathrm{A}$ " subunit and one dimer vacancy in each unit cell, while the $\gamma$ - $(4 \times 4)$ reconstruction is obtained by replacing only half of the "B" subunits ( $\mathrm{Si}=\mathrm{Si}$ dimers) with dimer vacancies, leading to a larger unit cell containing two "A" subunits, one "B" subunit, and one dimer vacancy. We expect that second-layer $\mathrm{Si}$ atoms exposed at dimer vacancies in the $\gamma$ - $(4 \times 4)$ reconstruction are rebonded as on clean $\mathrm{Si}(001)$ surfaces ${ }^{13,14}$ and have included these bonding interactions in fig. 4.

The differences in appearance between the boron-bonded Si dimers of the "A" subunit and the silicon-bonded dimers of the "B" subunits are attributed to a combination of structural and electronic effects.

Calculations for $\mathrm{SiH}_{3} \mathrm{BH}_{2},\left(\mathrm{SiH}_{3}\right)_{2} \mathrm{BH}$, and $\left(\mathrm{SiH}_{3}\right)_{3} \mathrm{~B}^{15}$ and experimental measurements for the $\operatorname{Si}(111)-(\sqrt{3} \times \sqrt{3})$ B reconstruction 9,10 and for $\delta$ doped $\mathrm{B} / \mathrm{Si}(001)$ layers $^{8}$ indicate $\mathrm{Si}-\mathrm{B}$ bond lengths of 2.0-2.1 $\AA$, substantially shorter than the $2.35 \AA$ bond length in bulk silicon. Thus, boron substitution at the $\mathrm{Si}(001)$ surface will distort the Si lattice to achieve a short B-Si bond. In our model, a shortened B-Si bond can be achieved by breaking the weak $\pi$-bond of the boron-bonded Si dimers; this might explain why the "A" features fail to show the splitting at positive bias that is characteristic of $\pi$-bonded dimers. Breaking the $\pi$ bond would also facilitate electron transfer from silicon to boron, in agreement with tunneling spectroscopy and SPV measurements. Filled-state STM images then likely reflect the position of the $\mathrm{B}-\mathrm{Si}$ backbonds and possibly the 
fourth $\mathrm{sp}^{3}$-hybridized boron dangling bond which, normally empty, could be partially filled due to charge transfer from Si. These dangling bonds on adjacent boron atoms might further interact to form B-B $\sigma$-bonds (not depicted in fig. 4). The above interpretation also explains why in filled state images the "A" features appear lower than the "B" features even though the "A" features show a high state density just below $E_{F}$ : the STM images contain both geometric and electronic information, and although the "A" features have a high state density just below $E_{F}$, the B-Si and partially-filled Boron "dangling bonds" are geometrically lower than the "B"-type Si=Si dimers.

The self-consistency of our model can be checked by measuring the areas of the reconstructed islands and terraces. Each $\alpha-c(4 \times 4)$ unit cell will eject six $\mathrm{Si}$ atoms if formed in the substrate plane, but requires addition of two $\mathrm{Si}$ atoms to form as an island. If this is the primary B-induced reconstruction then according to our model at high boron exposure the islands should occupy $75 \%$ of the surface area. Although a quantitative measurement is complicated by multiple reconstructions, our measurements do show that at high exposure the reconstructed islands occupy approximately $75 \%$ of the total surface area. At low exposure, STM images show only the "A" units, which constitute the $\beta$-c $(4 \times 4)$ reconstruction. In our model forming an " $A$ " unit as an "island" structure does not require any net gain or loss of Si. The extra Si required to form $\alpha-c(4 \times 4)$ and $\gamma-(4 \times 4)$ islands most likely originates by detachment from steps or by ejection from the terraces during boron incorporation; then, conditions that favor low $\mathrm{Si}$ adatom concentration (low temperature, low exposure) should favor the formation of the $\beta-c(4 \times 4)$ reconstruction, in agreement with our results. Our results also suggest that the ordering may 
be strongly dependent on the Si adatom density during diborane exposure.

Finally, our results are generally consistent with previous studies of $\delta$-doped $\mathrm{B} / \mathrm{Si}(001)$ layer formation using other precursors. ${ }^{1-8}$ Although these previous studies have shown $(2 \times 1)$ diffraction patterns in lowenergy electron diffraction (LEED) $)^{1,3-5}$, the arrangement of $\mathrm{Si}=\mathrm{Si}$ dimers and vacancies we observe in the STM typically leads to domain sizes of only 20-30 $\AA$. Since LEED samples surface order over a lengths of $>100 \AA$, only the shorter-range $(2 \times 1)$ periodicity is observable in this technique. In summary, our investigations have shown that the interaction of boron with $\mathrm{Si}(001)$ is strongly heterogeneous, producing regions of high boron concentration separated by regions of nearly clean Si. Based on the experimental data we have developed atomistic model of the structure and bonding of boron on the $\mathrm{Si}(001)$ surface and the formation of twodimensional "delta-doped" layers. Our work provides new insight into structure, bonding, and electronic properties of the boron-silicon system.

This work is supported in part by the U.S. Office of Naval Research and the National Science Foundation. The authors gratefully acknowledge the assistance of E.R. Frank and X. Chen.. 


\section{References:}

${ }^{1}$ M.L. Yu, D.J. Vitkavage and B.S. Meyerson, J. Appl. Phys. 59, 4032 (1986).

${ }^{2}$ B.S. Meyerson, F.K. LeGoues, T.N. Nguyen and D.L. Harame, Appl. Phys. Lett. 50, 113 (1987).

${ }^{3}$ R.L. Headrick, B.E. Weir, A.F.J. Levi, D.J. Eaglesham and L.C. Feldman, Appl. Phys. Lett. 57, 2779 (1990).

${ }^{4}$ R.L. Headrick, B.E. Weir, A.F.J. Levi, B. Freer, J. Bevk and L.C. Feldman, J. Vac. Sci. Technol. A 9, 2269 (1991).

${ }^{5}$ R.L. Headrick, B.E. Weir, A.F.J. Levi, D.J. Eaglesham and L.C. Feldman, J. Crystal Growth 111, 838 (1991).

${ }^{6}$ D.D. Koleske, S.M. Gates and D.B. Beach, J. Appl. Phys. 72, 4073 (1992).

${ }^{7}$ B.E. Weir, B.S. Freer, R.L. Headrick, D.J. Eaglesham, G.H. Gilmer, J. Bevk and L.C. Feldman, Appl. Phys. Lett. 59, 204 (1991).

${ }^{8}$ B.E. Weir, R.L. Headrick, Q. Shen, L.C. Feldman, M.S. Hybertsen, M. Needels, M. Schluter and T.R. Hart, Phys. Rev. B 46, 12861 (1992).

${ }^{9}$ R.L. Headrick, I.K. Robinson, E. Vlieg and L.C. Feldman, Phys. Rev. Lett. 63, 1253 (1989).

${ }^{10}$ E. Kaxiras, K.C. Pandey, F.J. Himpsel and R.M. Tromp, Phys. Rev. B 41, 1262 (1990).

${ }^{11}$ S.M. Gates and D.D. Koleske, Appl. Phys. Lett. 61, 309 (1992).

${ }^{12}$ C.P. Parry, R.A. Kubiak, S.M. Newstead, T.E. Whall and E.H.C. Parker, J. Appl. Phys. 71, 118 (1992).

${ }^{13}$ K.C. Pandey. in Proceedings of the Seventeenth International Conference on the Physics of Semiconductors (eds. Chadi, J.D. \& Harrison, W.J.) (Springer-Verlag, New York, 1985).

${ }^{14}$ R.J. Hamers, R.M. Tromp and J.E. Demuth, Phys. Rev. B 34, 5343 (1986).

${ }^{15}$ C.W. Bock, M. Trachtman and G.J. Mains, J. Phys. Chem. 89, 2283 (1985). 


\section{Figure Captions:}

Fig. 1) STM image of diborane-exposed surface showing formation of reconstructed islands and patchy reconstruction of substrate terrace. $\mathrm{V}_{\text {sample }}=+2.0 \mathrm{~V} ; I_{\text {tunnel }}=0.2 \mathrm{nA}$.

Fig. 2) High-resolution images of boron-induced reconstructions on $\mathrm{Si}(001)$ surface. $\alpha, \beta$, and $\gamma$ denote $\alpha-c(4 \times 4), \beta-c(4 \times 4)$, and $\gamma-(4 \times 4)$ unit cells of observed reconstructions; $A$ and $B$ denote structural subunits of reconstructions, as described in text..

a) High-resolution image showing boron-induced reconstruction and region of clean $\mathrm{Si}(001)-(2 \times 1)$, with $(2 \times 1)$ unit cell outlined.

Image area $108 \AA \times 152 \AA ; V_{\text {sample }}=-2.0 \mathrm{~V} ; I_{\text {tunnel }}=0.2 \mathrm{nA}$.

b) High-resolution imaging of occupied surface electronic states.

Image area $104 \AA \times 157 \AA ; V_{\text {sample }}=-2.0 \mathrm{~V} ; I_{\text {tunnel }}=0.2 \mathrm{nA}$.

c) Image showing identical region as (b), but tunneling into unoccupied electronic states.

Image area $104 \AA \times 157 \AA ; V_{\text {sample }}=+2.0 \mathrm{~V} ; I_{\text {tunnel }}=0.2 \mathrm{nA}$.

Fig. 3) Local tunneling spectroscopy measurements acquired above the "A" and "B" structural subunits. Curves have been displaced vertically for clarity.

Fig. 4) Structural sub-units of boron-induced reconstructions, and proposed arrangements of these subunits to form $\alpha-c(4 \times 4), \beta-c(4 \times 4)$, and $\gamma-(4 \times 4)$ unit cells. 


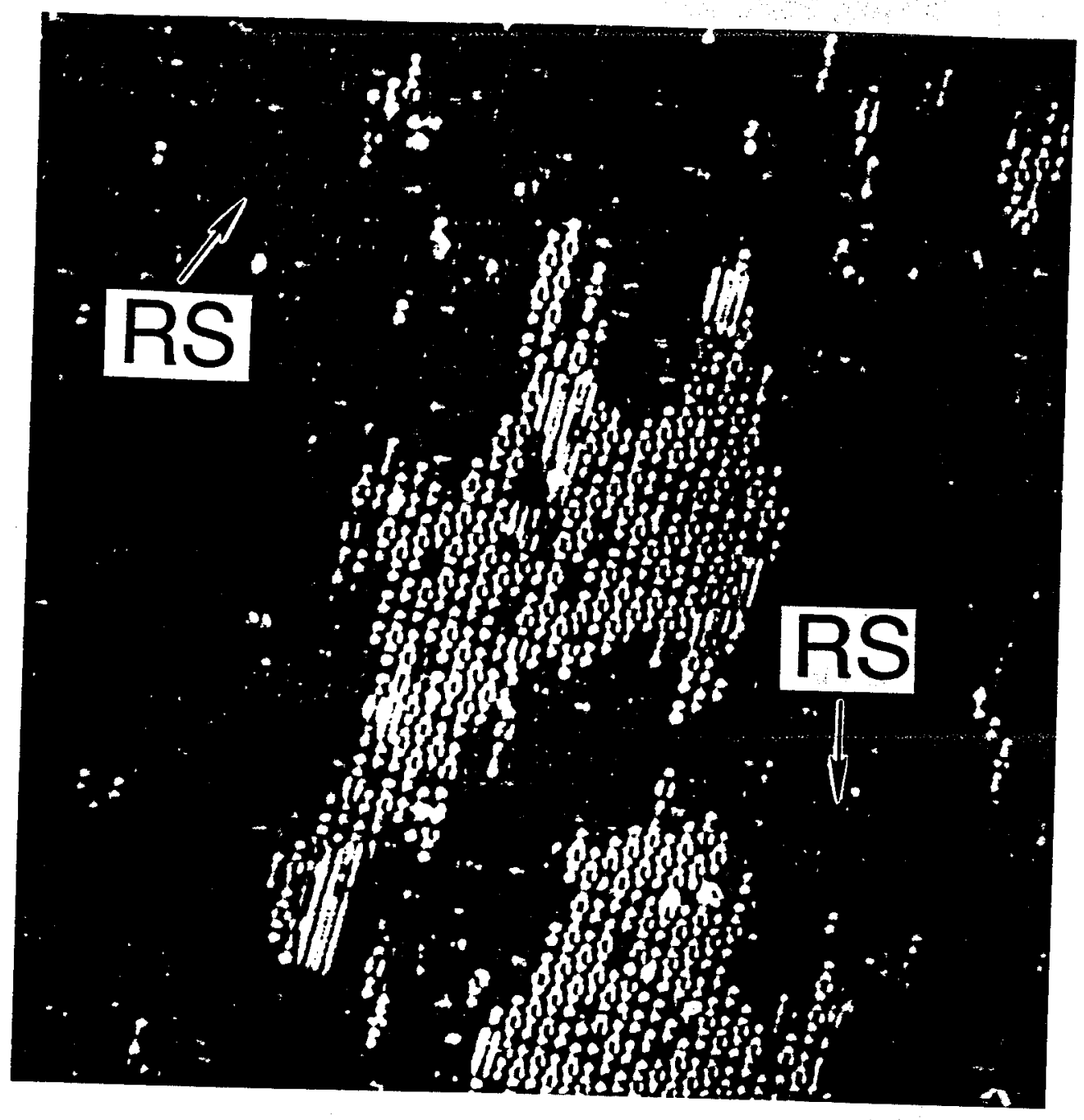

Wing, Hamers and Kaxiris
PRL

Fis. 1 


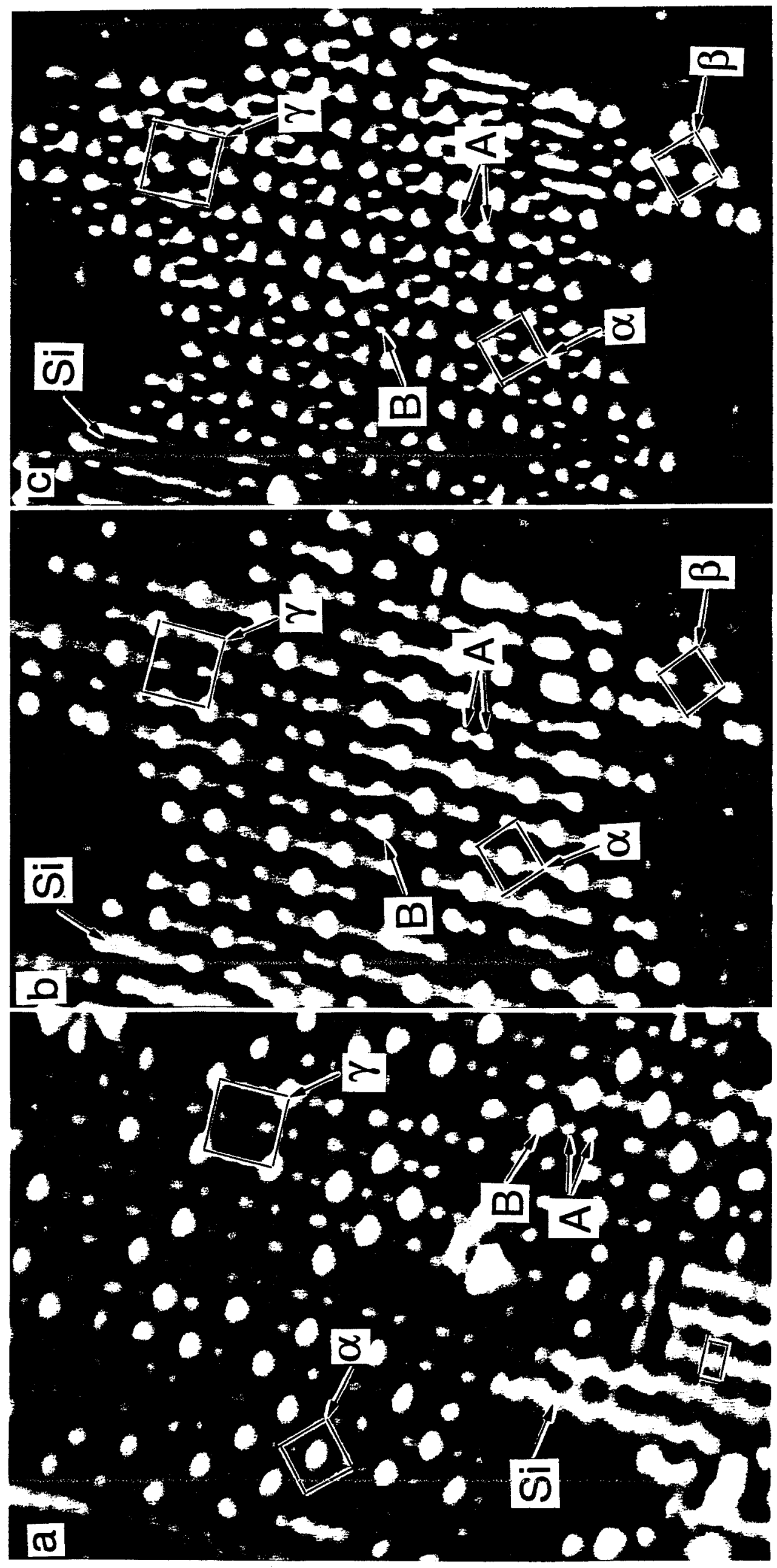

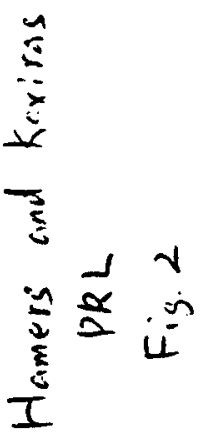

$\sum_{3}^{5}$ 


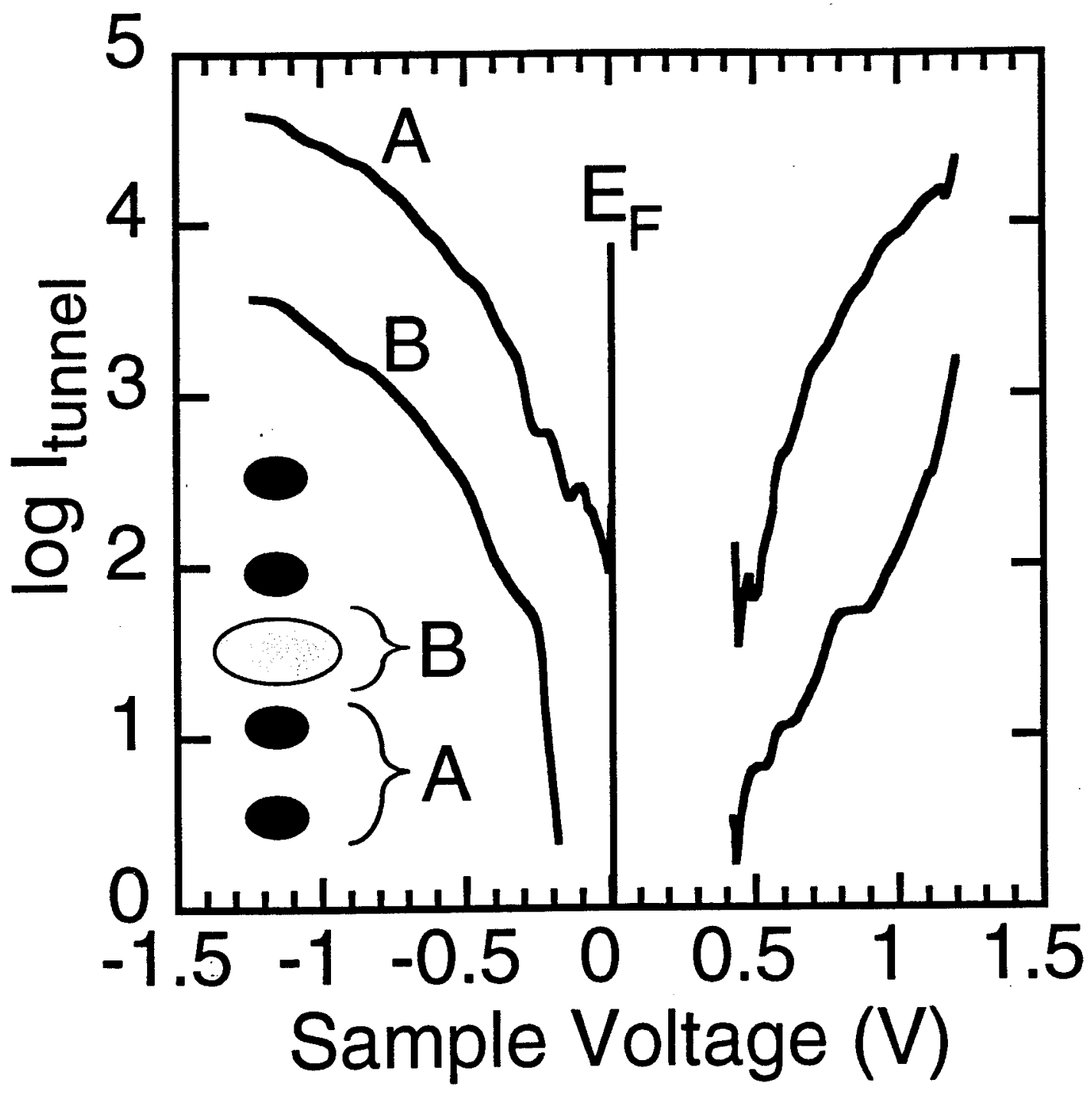

Waing, Hamers and Kaxirus PRL

Fis. 3 


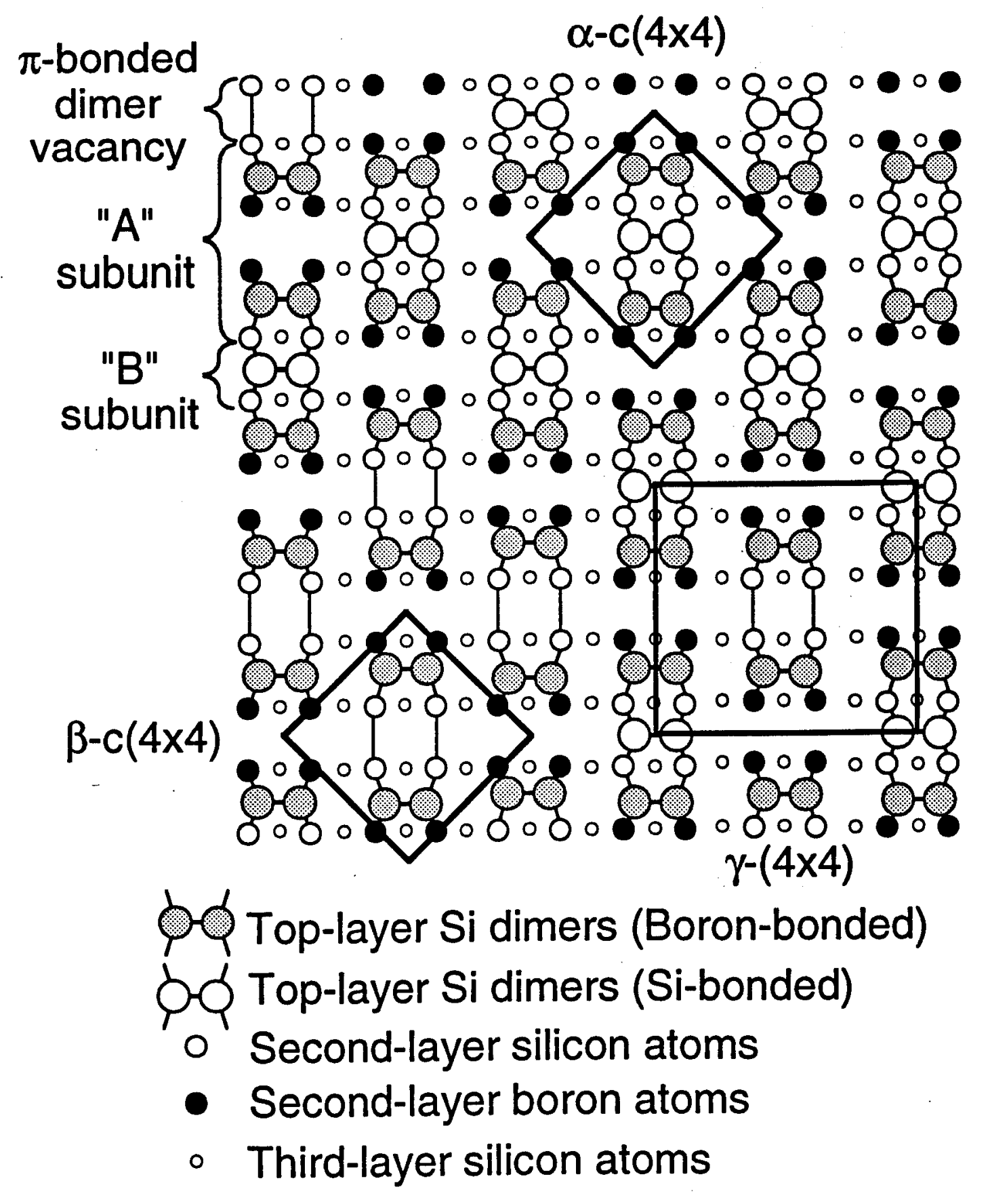

Wang, Hamers, and Kaxiras PRL

Figure 4 